\section{A case of malignant spinal cord ependymoma in association with a duplication of part of the long arm of chromosome 12}

A 12 year old girl of Greek Cypriot origin presented with progressive gait disorder. There was no relevant family history. Her early development was rather slow and she made poor progress at a normal school. An assessment using the WISC had given her a full scale IQ of 65 . Her early walking was reported as having a stamping quality tending to drag the left leg. However, no further problem was noticed until $2 \frac{1}{2}$ weeks before admission, when her gait had deteriorated progressively.

On admission she was found to be microcephalic with a head circumference of $47.5 \mathrm{~cm}$, which is more than $-3 \mathrm{SD}$ below the mean. There were some dysmorphic features: arachnodactyly, hypermobile joints, proximally placed thumbs, and immobile first metacarpal-phalangeal joints. Forced eye closure produced an appearance reminiscent of a whistling face. The peripheral circulation was poor. There was marked weakness of the legs, in the left more than the right, with pathologically brisk tendon reflexes and bilateral extensor plantar responses, but she was still able to walk. In the upper limbs there was a mild degree of

Received for publication 9 May 1984.

Accepted for publication 24 July 1984.

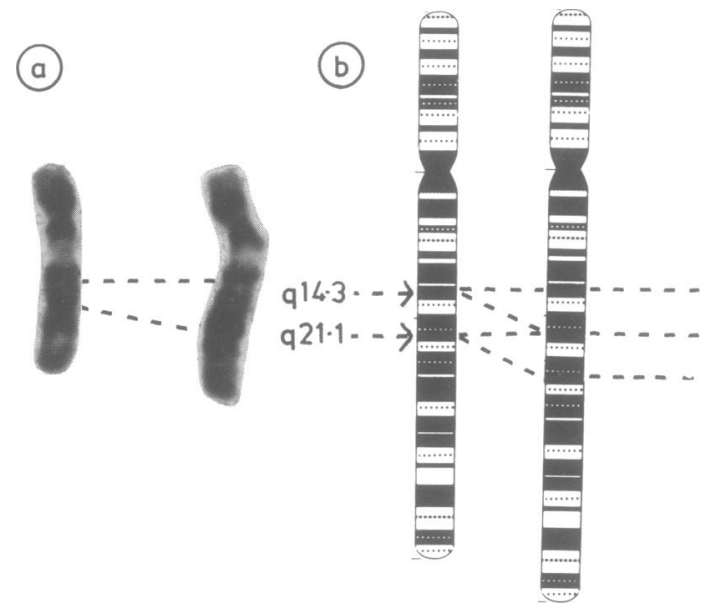

FIGURE (a) Partial karyotype showing the two chromosomes 12. The increase in length of the abnormal chromosome (shown on the right) between the bands q14.3 and 921.1 is thought to be due to a duplication. (b) Idiogram showing the position of the breakpoints and the region of duplication. pyramidal weakness, on the left more than the right, and an intention tremor. Sensory testing was difficult because of her poor cooperation. A CT scan was normal. A myelogram showed a large intramedullary tumour in the upper thoracic and cervical region. The CSF protein was markedly raised.

A laminectomy was performed by Mr Charles Polkey at $\vec{\omega}$ the Guy's/King's/Maudsley Neurological Unit. Histology showed a malignant ependymoma, in which most of the cells had a perivascular concentration. The cells had moderately generally hyperchromatic nuclei and mitotic N figures were present. She was then treated with a iv combination of steroids and radiotherapy with some $\vec{G}$ improvement, but 8 months later her gait again deterio- $\rightarrow$ rated. A sub-total excision of tumour was then performed, 음 which was followed by transient improvement. A year from the first presentation she developed raised intra- $D$ cranial pressure. A CT scan showed a mass in the brain stem and severe hydrocephalus. She died shortly after this and necropsy was not performed.

During the patient's first admission cytogenetic studies of on peripheral blood cultures were carried out. Giemsa banded chromosome preparations showed an abnormality of chromosome 12, which was interpreted as a possible duplication of band $q 15$, together with parts of the two adjacent bands (q14.3 and $\mathrm{q} 21 \cdot 1$ ), in which case her chromosome complement would be written: $\triangle$ $46, X X, \operatorname{dup}(12)(q 14 \cdot 3 \rightarrow q 21 \cdot 1)$.

However, more than one interpretation is possible and $\frac{\mathrm{O}}{3}$ instead of a duplication, the abnormality could be the result of an interstitial insertion of chromosome material of $\frac{\overrightarrow{7}}{0}$ unknown origin.

We are grateful to Dr B Dunkley for the histology report.

B G R Neville, i

A C BERRY, AND Y STODDART 2

Newcomen Centre and $\frac{\mathrm{O}}{3}$ South East Thames Regional Genetics Centre, Guy's Hospital, 음 London SE1 9RT. I

Correspondence and requests for reprints to $\mathrm{Dr} A \mathrm{C}$. Berry, SE Thames Regional Genetics Centre, Paediatric ${ }^{N}$ Research Unit, Guy's Tower, 8th Floor, London SE1 9RT.

\section{Interstitial deletion of chromosome 2}

The proband described here was the first child of a 26 year $\square$ old mother and 29 year old father. The mother had had $\bar{D}$ one spontaneous abortion in the fourth month of a $\overrightarrow{\mathbb{D}}$ previous pregnancy. There was no family history of $\frac{\rho}{\mathbb{Q}}$ Received for publication 13 March 1984. Accepted for publication 10 June 1984. 